\section{PSICOLOGÍA IBEROAMERICANA}

\section{Psicología lberoamericana}

ISSN: 1405-0943

psicología.iberoamericana@uia.mx

Universidad Iberoamericana, Ciudad de

México

México

Acosta López de Llergo, Irma Cristina; Sánchez Araiza, Yamna Idalia Manifestación de la Resiliencia como Factor de Protección en Enfermos Crónico Terminales Hospitalizados

Psicología Iberoamericana, vol. 17, núm. 2, julio-diciembre, 2009, pp. 24-32

Universidad Iberoamericana, Ciudad de México

Distrito Federal, México

Disponible en: http://www.redalyc.org/articulo.oa?id=133912609004

- Cómo citar el artículo

- Número completo

- Más información del artículo

Página de la revista en redalyc.org

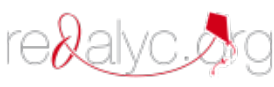

Sistema de Información Científica

Red de Revistas Científicas de América Latina, el Caribe, España y Portugal

Proyecto académico sin fines de lucro, desarrollado bajo la iniciativa de acceso abierto 


\title{
Manifestación de la Resiliencia como Factor de Protección en Enfermos Crónico Terminales Hospitalizados
}

\author{
Resilience Manifestation as a Protection Factor Among \\ Chronic Terminal Hospitalized Patients
}

Irma Cristina Acosta López de Llergo ${ }^{1}$

Yamna Idalia Sánchez Araiza

Universidad Iberoamericana, CD. de México, México

\section{RESUMEN}

En la actualidad los profesionales de la salud encuentran que las enfermedades crónico terminales son consideradas como fuentes generadoras de estrés que implican un proceso de deterioro continuo. Sin embargo, la mayoría de las personas que padecen una enfermedad terminal no sólo enfrentan la situación sino que en algunas ocasiones la superan saliendo enriquecidos por la experiencia. El presente estudio tiene como objetivo describir los factores de protección que favorecen la resiliencia en los pacientes crónico terminales internados en el Hospital General Regional No. 1 del Instituto Mexicano del Seguro Social (IMSS). Se realizó una investigación cualitativa en donde se llevaron a cabo entrevistas a profundidad con enfoque clínico con una muestra de 213 personas. Se plantearon tres categorías que engloban las áreas de los factores de protección (individual, familiar y la comunidad). Se concluye que la orientación psicológica y la resiliencia deben promover un enfoque basado en las fortalezas y recursos del individuo para disminuir los factores de riesgo y eventos estresantes.

Descriptores: Enfermedad crónico terminal, resiliencia, orientación psicológica.

\section{ABSTRACT}

Health professionals have found nowadays that terminal chronic diseases are considered sources of stress that entail a process of continuous deterioration. However, most people who suffer from a terminal illness not only face up the situation, but sometimes they go through it and emerge enriched by that experience. The present study aimes to describe the protection factors that promote the resilience in terminal-chronic patients hospitalized at the Hospital General Regional No. 1 of the Instituto Mexicano del Seguro Social (IMSS). A qualitative study was conducted in which in-depth interviews with a clinical approach were carried out with a sample of 213 persons. Three categories that encompass the areas of protective factors (individual, family and community) were established. It can be concluded that counseling and resilience should promote an approach based on the strengths and resources of the individual in order to reduce risk factors and stressful events.

Key words: Terminal chronic disease, resilience, counseling.

\footnotetext{
${ }^{1}$ Para correspondencia: Cristina Acosta, Dirección: Plaza del Duomo No. 5-A, Fraccionamiento Lomas Boulevares, Tlalnepantla de Baz, C.P. 54020, Edo. de México. Tel.: (52) (55) 53704684 y 53707199. Cel.: 0445515122138. Correo electrónico: cris_allergo78@hotmail.com. Yamna Sánchez, Dirección: Calle Pavo Real No. 25, Mayorazgos del Bosque, Arboledas, Atizapán de Zaragoza, C.P. 52957, Edo. de México. Tel.: (52) (55) 53799453, cel.:0445532693832, yamnis@hotmail.com.
} 


\section{INTRODUCCIÓN}

A lo largo de la historia, la psicología centró su atención en la patología de los individuos que, generalmente, incidía en las áreas que dificultaban que el sujeto se encontrara integralmente sano (Seligman y Csikszentmihalyi, 2000). Esto ha cambiado gracias al surgimiento de la psicología positiva y el enfoque que Rogers (1997) muestra sobre la visión de la persona, en la que resalta sus recursos, fortalezas y le brinda aprecio positivo incondicional.

Algunas de las recientes investigaciones (Kern \& Moreno, 2007; Martorelli \& Mustaca, 2004; Suárez Ojeda \& Melillo, 2001; Szarazgat \& Glaz, 2006; Vera Poseck, Carbelo \& Vecina, 2006) se han interesado por conocer la manera en que los individuos enfrentan exitosamente la adversidad y las diferentes crisis que atraviesan en su desarrollo, cómo y qué es lo que aprenden de tales experiencias.

Es así como, gracias al paido-psiquiatra Michael Rutter (Vera Poseck et al., 2006) surge el concepto de resiliencia en el ámbito psicológico en la década de 1970, definido como la capacidad de un individuo de reaccionar ante adversidades y recuperarse. Esto implica un conjunto de cualidades que fomentan un proceso de adaptación exitosa y transformación a pesar de los riesgos (Suárez Ojeda \& Melillo, 2001; Szarazgat \& Glaz, 2006). Grotberg (1997) menciona que es una capacidad propia del ser humano mientras que otros autores coinciden que es un proceso dinámico con una amplia combinación de factores de riesgo y protección, producto de una conjunción entre el ambiente y el individuo.

El enfoque de la resiliencia entiende el desarrollo humano dentro de un contexto específico, es decir, si cada individuo está inmerso en un marco ecológico, entonces, para comprender mejor el proceso de resiliencia, es de suma importancia considerar el ambiente y la cultura del individuo, al igual que las tareas específicas correspondientes a cada etapa de su desarrollo.

Kobasa, Maddi y Kahn (1982) mencionaron por primera vez el concepto de "personalidad resiliente" en relación con la idea de protección frente a los factores que provocan estrés. Es así que establecieron que las personas con características resilientes presentan un gran sentido del compromiso, una fuerte sensación de control sobre los acontecimientos, están más abiertos a los cambios, a la vez que tienden a interpretar las experiencias estresantes y adversas como una parte más de la vida. Estos autores consideran la "personalidad resiliente" como un constructo multifactorial con tres componentes principales: compromiso, control y reto.

Es importante resaltar que la resiliencia puede ser fomentada, es decir, es una capacidad universal pero no es absoluta ni estable, por lo que una persona puede ser resiliente en un medio y no en otro (Kern \& Moreno, 2007). Así, la teoría de la resiliencia cobra significado a partir de las diferencias en la reacción ante circunstancias adversas, generadoras de estrés. Mientras algunas personas sucumben ante dichas situaciones, evidenciando desequilibrio y trastornos a diversos niveles, otras fomentan un proceso de adaptación exitosa y transformación a pesar de la adversidad.

El proceso de desarrollo por sí mismo constituye un modelo de adaptación a través del tiempo (Yates, Egeland \& Sroufe, 2003). Por eso, los niveles anteriores de adaptación son solamente probabilísticos y no determinantes del funcionamiento posterior. La competencia en cierto periodo evolutivo da al individuo los fundamentos para seguir con su desarrollo en las etapas subsecuentes. Por otro lado, la inadaptación puede comprometer la capacidad de la persona a un desarrollo posterior. De acuerdo con Yates y Zelazo (2003), la adaptación positiva se refiere a la adaptación sustancialmente superior a la esperada dada la exposición a circunstancias de riesgo (resiliencia). Lo anterior no significa necesariamente la eliminación del problema, sino la aparición de un nuevo contexto de significado y manejo del mismo.

El individuo no es un receptor pasivo de los diferentes estímulos, sino que los modula constantemente con su conducta. En este proceso participan componentes genéticos, psicológicos, sociales y culturales. De acuerdo con Kern y Moreno (2007), tanto los factores de riesgo como los de protección deben ser vistos como resultado de la estrecha interacción individuoambiente, donde cada una de estas partes juega un papel activo; su manejo eficiente se da como resultado de la manifestación de la capacidad resiliente individual.

Un factor de riesgo son todas aquellas características o situaciones propias de la persona o de su entorno que aumentan la posibilidad de desarrollar desajuste y desequilibrio psicosocial. Son obstáculos que aumen- 
tan la vulnerabilidad en el desarrollo de la persona. Algunos ejemplos pueden ser: pobreza, problemas familiares, violencia, enfermedades, pérdidas, guerras, etcétera. Es importante no considerar estos factores de manera aislada sino también tomar en cuenta su interacción y proceso de desarrollo, ya que co-ocurren con otros factores de riesgo usualmente sincronizados con una secuencia de experiencias estresantes y no sólo de un evento único (Kern \& Moreno, 2007). El factor de protección se define conceptualmente como todas aquellas características o situaciones propias de la persona o de su entorno que elevan su capacidad para hacer frente a la adversidad o disminuyen la posibilidad de desarrollar desajuste psicosocial aún con la presencia de factores de riesgo (Masten \& Powell, 2003; Place, Reynolds, Cousins \& O’Neill, 2002). Los autores citados identificaron un gran número de factores de protección ante la adversidad y los agruparon en tres áreas: individuales, familiares y de la comunidad.

Dentro de los factores individuales se incluyen características temperamentales que provocan respuestas positivas. Algunas de las más importantes son: autoestima elevada, habilidades comunicativas, habilidad para la resolución de problemas, capacidad de introspección, creatividad, independencia, capacidad de relacionarse estableciendo lazos afectivos, iniciativa, sentido del humor, curiosidad por conocer disfrutando de sus experiencias, adecuada tolerancia a la frustración, responsabilidad, estrategias de afrontamiento y competencias cognitivas. En el caso de los familiares, lo integran características relacionadas con la cohesión, ternura, sentimiento de un vínculo afectivo fuerte por parte de los progenitores, preocupación por el bienestar, presencia de apoyo incondicional, fomento a creencias religiosas, seguridad y estabilidad emocional proporcionada por un modelo parental adecuado.

Finalmente, en el ámbito comunitario se consideran características como la presencia de pares, redes de apoyo adecuadas (amigos y trabajo), participación en un grupo religioso, conexión con organizaciones en pro de la sociedad, y calidad del entorno, así como los servicios sociales y de salud. Cabe mencionar que los factores de protección, al igual que los de riesgo, no actúan aisladamente en el individuo, sino que ejercen un efecto conjunto en el que se establecen complejas relaciones funcionales que traen como consecuencia el debilitamiento de los efectos de las circunstancias adversas y eventos estresantes. Este proceso es denominado por Fraser (1997) como "moderación del estrés" y nos permite comprender los mecanismos que subyacen a los factores de protección y a la resiliencia en general.

En el ámbito de la salud, las investigaciones sobre la resiliencia han cambiado la forma en que se percibe al ser humano: de un modelo de riesgo basado en las necesidades y en la enfermedad, se ha pasado a un modelo de prevención y promoción basado en las fortalezas y recursos que el ser humano tiene en sí mismo y a su alrededor (Cyrulnik, 2002).

El hombre toma elementos para mantener su equilibrio en la vida y cuando alguno de estos elementos no se tiene en la cantidad y calidad adecuadas, aparece un estado de necesidad o desequilibrio en donde se busca recuperar el equilibrio inicial. Si las acciones para recuperar esto no se realizan o no son suficientes, este desequilibrio puede llevar al individuo a la pérdida del estado de salud (Calhoun \& Tedeschi, 2006).

Así, la enfermedad es la pérdida del equilibrio entre cuerpo, mente y ambiente, ocasionado por la insatisfacción de alguna necesidad.

La enfermedad posee una característica que es la singularidad, es decir, la actitud del paciente frente a la enfermedad es un factor caracterológico del enfermo mismo. Se halló que la buena salud objetiva guarda escasa relación con la sensación de bienestar. El optimismo, un sentido de control personal y la habilidad para encontrar significado a las experiencias de la vida se asocian con una mejor salud mental. Importa más la percepción subjetiva del estado de salud para salir adelante y afrontar mejor la situación presente (Martorelli \& Mustaca, 2004), de tal suerte que la psicología de la salud tiene como uno de sus objetivos la promoción y el mantenimiento de la salud, la prevención y el tratamiento de la enfermedad.

En el caso de los enfermos crónico terminales, se considera a la resiliencia como un punto fundamental ya que el paciente al ser diagnosticado se enfrenta a situaciones desfavorables en las áreas emocional, social, familiar y física. Es por ello que el equipo de salud debe hacerlos conscientes de sus recursos, aquellos que les permitan sentirse como un ser humano con posibilidades óptimas para continuar su vida a pesar de la 
situación crítica por la que atraviesa y obtener un beneficio del nuevo estilo de vida que va a implementar (Kern \& Moreno, 2007). Los orientadores psicológicos no deben dejar de lado los recursos y fortalezas de los individuos, sino más bien reforzarlos constantemente para así fomentar la resiliencia como un proceso determinante de la salud integral.

El estudio de la resiliencia relacionada con la enfermedad crónica significa un cambio de perspectiva en el campo de la salud, pues enfoca el desarrollo positivo del individuo (Kern \& Moreno, 2007).

En la actualidad, las enfermedades crónicas son consideradas como fuentes generadoras de estrés, ya que implican un proceso de deterioro continuo y constante de varios años que afecta la calidad de vida de la persona, generando nuevas situaciones para afrontar. El tratamiento médico para ellas se ha desarrollado mucho en los últimos años y algunos estudios en psicología han enfocado su impacto sobre el bienestar y la salud mental de la persona (Eiser, 1996; Garralda, 1994; Perrin \& Shonkoff, 2000).

Se debe tomar en cuenta que tanto personas sanas como enfermas pasan por las mismas etapas de desarrollo, pero para estas últimas es más complicado debido a la influencia de los aspectos fisiológicos de la propia enfermedad, el tratamiento, las hospitalizaciones, limitaciones y alteraciones en las relaciones familiares (Midence,1994).

Aley (2002) menciona que el papel de los profesionales de la salud es ayudar a la persona enferma a tener un desarrollo óptimo dentro de las propias limitaciones dadas por su condición, es decir, no sólo atender la parte física sino ver a la persona en su totalidad, no sólo su enfermedad.

La adaptación del paciente a la enfermedad crónica se da por medio de la relación entre los factores de riesgo y de protección. En los factores de riesgo hay tres características esenciales: variables de la enfermedad (gravedad, visibilidad y problemas médicos asociados), nivel de funcionamiento independiente $y$ estresores psicosociales que se relacionan con la enfermedad. Entre los factores de protección están las variables intrapersonales (temperamento, habilidad para resolver problemas, etcétera), ecología social (ambiente familiar, apoyo social, recursos de la comunidad) y habilidades en el procesamiento del estrés (evaluación cognitiva y estrategias de afrontamiento) (Bradford, 1997).

Bennet (1994) considera que los trastornos psicológicos de los enfermos crónicos se deben, en parte, a sus pocas oportunidades de socialización, a su extrema dependencia a sus familiares y al equipo de salud y a sus limitaciones físicas.

Por lo tanto, el objetivo del presente trabajo es describir los factores de protección que favorecen la resiliencia frente a situaciones adversas en pacientes hospitalizados para promover la mejora en cuanto a la asistencia de necesidades que presentan, de forma que les resulte más fácil desarrollar características resilientes si existen condiciones personales y ambientales favorables.

\section{MÉTODO}

\section{Resiliencia como apoyo psicológico}

Cabe mencionar que esta investigación retoma datos de un trabajo anterior más amplio en donde el tema principal fue conocer la opinión que tenían los pacientes en cuanto a la relación con su médico dentro del hospital. El escenario donde se realizo el levantamiento de datos fue el Hospital General Regional (HGR) No. 1 del Instituto Mexicano del Seguro Social (IMSS), Dr. Carlos MacGregor Sánchez Navarro (antes Hospital Gabriel Mancera), considerado de segundo nivel. Atiende más de 3 mil consultas de urgencias al mes en sus unidades médicas siendo los traumatismos, las infecciones respiratorias, gastrointestinales, diabetes mellitus y sus complicaciones los más atendidos en el Instituto.

\section{Participantes}

La muestra estuvo formada por 213 enfermos crónico terminales hospitalizados cuyas edades oscilaron entre los 17 y 97 años. El 51\% fueron hombres y el $49 \%$ fueron mujeres. El porcentaje por diagnóstico médico fue: diabetes $14.6 \%$, insuficiencia renal $21.6 \%$, enfermedades cerebro vasculares $8.5 \%$, neuropatía $10.8 \%$, cáncer $1.9 \%$, VIH $1.4 \%$, cardiopatías $14.6 \%$, enfermedades hepáticas $6.6 \%$, otras $18.3 \%$ y enfermedades en estudio $1.9 \%$, tomando en cuenta el diagnóstico de la enfermedad principal aunque muchas veces el paciente presentaba complicaciones referentes a la misma. 


\section{Instrumento}

Se diseñó una entrevista a profundidad con enfoque clínico en donde se evaluaron dos aspectos:

1. El funcionamiento mental: a) Orientación, b) Curso de pensamiento, c) Contenido de pensamiento y d) Sensopercepción

2. El funcionamiento emocional: a) Emociones, b) Actitud hacia la enfermedad, c) Área de mayor dificultad, d) Recursos y fortalezas y e) Descripción de la orientación psicológica.

En la presente investigación se llevó a cabo un estudio descriptivo de tipo cualitativo en el cual se analizaron los recursos y las fortalezas que el paciente mostró como factores protectores que promueven la resiliencia.

\section{Procedimiento}

Se elaboró el formato de entrevista a profundidad para evaluar a pacientes crónico terminales hospitalizados que así lo consentían, donde cada una de ellas tenía un tiempo variable pero no menor a 30 minutos. La intervención que se ofrecía era de entre una y cinco sesiones, de acuerdo a la estadía de los pacientes en el hospital; el seguimiento se interrumpía en caso de cambio de área, fallecimiento o alta. Las entrevistas se registraron en el protocolo en donde se describían las fortalezas y los recursos mostrados por los pacientes. Los datos se acomodaron en categorías formando grupos con base en los diferentes recursos o fortalezas encontrados.

El equipo de orientación psicológica estuvo constituido por siete psicólogos, quienes rotaron tres diferentes áreas del hospital: Medicina Interna, Nefrología y Urgencias. El trabajo de campo fue realizado durante los meses de agosto a noviembre de 2007.

Es importante mencionar que el equipo de orientadores contó con la supervisión semanal tanto individual como grupal de una doctora en Psicología del mismo instituto de salud pública, quien brindó retroalimentación para mejorar la calidad de las intervenciones así como para desarrollar el óptimo funcionamiento de los orientadores, elaborando y analizando la transferencia y contratransferencia con los pacientes.

El análisis de la información se basa en la propuesta hecha por Masten \& Powell (2003) y Place et al. (2002).

\section{RESULTADOS}

Una vez aplicada la entrevista a profundidad, se identificaron las características por categoría (Tabla 1).

\section{FACTORES DE PROTECCIÓN INDIVIDUALES}

De acuerdo con la orientación psicológica que se dio a los enfermos crónico terminales dentro del IMSS, se pudo observar que presentaban diversos recursos o fortalezas como: alto nivel de espiritualidad, esperanza y una actitud positiva frente a su diagnóstico, lo cual los hacía ver de un modo distinto la situación o el pronóstico; sin embargo, no necesariamente mostraban sentido del humor o un autocuidado adecuado.

Una característica observada de manera constante en los enfermos fue la fortaleza reflejada en sus ganas de vivir y afrontar la situación adversa. Esto se podía notar, por ejemplo, en la actitud que presentaban conforme avanzaba la entrevista, lo cual les permitía darse cuenta de la situación de forma más objetiva y así actuar de manera responsable en el tratamiento y cuidados indicados por el médico para lograr la mayor independencia posible en cuanto a su persona.

De igual manera, se pudo notar que dentro de la orientación psicológica brindada, los enfermos manifestaron situaciones de crisis anteriores que resolvieron de forma satisfactoria. Es importante resaltar que este factor contiene dos características importantes, las herramientas y recursos utilizados y la resolución del conflicto en sí. Ambas generan una notable confianza en sí mismos que a su vez refuerza la autoestima para enfrentar factores de riesgo futuros. Tal es el caso de "Carmen R.", quien cambió de actitud en cuanto a su autocuidado después de que su esposo falleció a causa de la diabetes y ella tuvo que salir adelante con sus dos hijos, dándose cuenta de la situación en la que se encontraba, y que ella fue diagnisticada como prediabética. Mencionó que a partir de ahí se preocupó más por su salud y procuró cuidarse "lo mejor posible".

\section{FACTORES DE PROTECCIÓN FAMILIARES Y/O COMUNITARIOS}

El papel que juega la familia dentro del contexto de un enfermo crónico terminal es de gran importancia debido a que el apoyo recibido por parte de estos miembros impulsa al paciente a tomar mayor fortaleza para salir adelante y no sentirse solo frente a la situación 
Tabla 1. Categorías generadas a partir de la entrevista a profundidad diseñada para pacientes crónico terminales hospitalizados, siguiendo el modelo teórico de Masten y Powell (2003) y Place et al. (2002).

\begin{tabular}{|l|l|}
\hline \multicolumn{1}{|c|}{ Factores de Protección Individuales } & \multicolumn{1}{|c|}{ Factores de Protección Familiares y/o de la Comunidad } \\
\hline Autoestima adecuada & Red de apoyo (familia) \\
\hline Actitud positiva frente a la enfermedad & Vínculo afectivo fuerte \\
\hline Autocuidado & Red de apoyo (amigos) \\
\hline Espiritualidad & Trabajo (nuevos proyectos) \\
\hline Sentido del humor & Calidad en los servicios de salud recibidos \\
\hline Fortaleza (perseverancia y ganas de vivir) & Religión \\
\hline Capacidad reflexiva & Estilos de vida saludables \\
\hline Motivación & Relaciones sociales adecuadas \\
\hline Inteligencia emocional & Búsqueda de ayuda externa \\
\hline Esperanza & Calidad del entorno \\
\hline Actuar de manera Independiente & \\
\hline Capacidad intelectual & \\
\hline Resolución de crisis pasadas (estrategias de afrontamiento) & \\
\hline $\begin{array}{l}\text { Habilidades en el procesamiento del estrés (evaluación } \\
\text { cognitiva) }\end{array}$ & \\
\hline Salud mental adecuada & \\
\hline
\end{tabular}

Nota: Categorías de los factores de protección observados durante la orientación psicológica con pacientes crónico terminales hospitalizados en el Hospital General Regional No. 1 Dr. Carlos MacGregor Sánchez Navarro del Instituto Mexicano del Seguro Social en la ciudad de México

por la que atraviesa. Esto se apreció durante la investigación, ya que los pacientes veían como un aliciente el que sus familiares estuvieran presentes durante su estadía en el hospital y fuera de él, ya que éstos les brindan apoyo, atención, cuidados, confianza y compañía dentro de este proceso que puede llegar a ser tan doloroso. Un ejemplo de lo anterior puede ser la situación de "Esther G., quien a sus 77 años necesitaba cuidados constantes y continuos debido a su diabetes, por lo que sus cuatro hijos llegaron a un acuerdo en donde cada uno la tendría en su casa por algún tiempo determinado para continuar con el tratamiento una vez que saliera del hospital, lo cual la motivó a lograr su pronta recuperación.

Por la misma razón, contar con un vínculo afectivo fuerte y seguro de los familiares forma parte de un proceso que actúa como intermediario en los comportamientos resilientes de la persona enferma, como lo expone "Cristina M.": "Es la segunda vez que estoy hospitalizada pero me siento tranquila ya que mis hijos me visitan diario y no me han dejado sola, siento su apoyo y eso me ayuda a tener fuerza". No obstante, cabe recalcar que esta categoría considerada como independiente, resulto muy pequeña en estos pacientes, por lo que más bien se incluyó como un servicio más dentro de la categoría de factores de protección comunitarios, quizás porque la muestra estuvo constituida por pacientes hospitalizados.

El entorno social en el que se desenvuelve el paciente juega un papel significativo en el desarrollo de factores de protección resilientes, ya que las redes de apoyo alrededor de la persona, como son los amigos, el trabajo y la religión, se convierten en un fuerte apoyo durante el difícil proceso de la enfermedad.

Es así que la participación del enfermo en un grupo religioso puede reforzar los valores familiares e 
individuales brindándole paz, esperanza y cierto alivio como un elemento relevante para afrontar la situación por la que atraviesa, como es el caso de "Libertad M., quien practica el cristianismo y recurre a las oraciones para mantenerse fuerte y salir adelante, como ella misma menciona.

De la misma forma, se observó que los pacientes que planeaban nuevos proyectos de vida laboral, presentaban una mayor motivación para salir adelante y esforzarse por conseguir nuevas metas.

Por lo tanto, la calidad del entorno también influía en su decisión de buscar apoyo o ayuda externa, ya fuera en centros de apoyo, psicólogos o especializados en determinada enfermedad, lo cual a su vez fomentaba estilos de vida más saludables dentro de su propio contexto, como lo menciona "Aaron C., quien sentía culpa por tomar bebidas alcohólicas en exceso, motivo por el cual fue hospitalizado, ya que sentía que "eso era mas fuerte que él". Sin embargo, después de la orientación se comprometió con él mismo y con su hermana ahí presente en buscar un grupo de ayuda que lo apoyara para "salir adelante".

La atención recibida por parte del servicio hospitalario (relación médico-paciente) fue un factor primordial que influyó en el estado de ánimo del paciente y en sus expectativas en cuanto a la enfermedad. Los pacientes manifestaron que es fundamental que el equipo de salud les proporcione un trato amable, de respeto y confianza, brindándoles información veraz en cuanto a su padecimiento.

Es importante mencionar que ninguna de estas tres categorías es determinante por sí misma, ya que la interacción entre lo individual, familiar y comunitario promueve un mayor número de recursos y fortalezas en el individuo, lo que indica que el proceso de resiliencia se caracteriza por su naturaleza dinámica.

La diversidad de los resultados mostrados en función al nivel de resiliencia manifestado y el manejo del diagnóstico depende en gran medida de la propia condición física y limitaciones de la enfermedad pero también de los factores personales, familiares y sociales de los cuales disponen los individuos.

\section{DISCUSIÓN Y CONCLUSIONES}

Las enfermedades crónicas son un grupo heterogéneo de dolencias con distintas características. En su mayor parte son progresivas, causan un deterioro físico importante, perjudican el desarrollo y requieren tratamientos dolorosos y largas hospitalizaciones. El tratamiento médico para estas enfermedades se ha desarrollado mucho en los últimos años, pero pocos estudios en psicología han enfocado su impacto sobre el bienestar y la salud mental. Siguiendo la misma línea, el estudio de la resiliencia es relativamente reciente en psicología, y más aún cuando se aplica a la enfermedad crónica como un factor de riesgo en el desarrollo. Significa un cambio de perspectiva importante, ya que enfoca el desarrollo positivo, la posibilidad de superación y la adaptación frente a la enfermedad (Kern \& Moreno, 2007). Sin duda, los programas de salud mental en el contexto hospitalario pueden ayudar a los enfermos crónicos a una mejor adaptación a su dolencia. Sin embargo, el desarrollo de la resiliencia todavía representa un desafío para los investigadores y para una aplicación práctica de los conocimientos ya adquiridos.

Como se pudo notar en la presente investigación, la población estudiada reveló características resilientes como lo fueron un gran sentido del compromiso, la sensación de control sobre los acontecimientos y una gran capacidad de reto (Kobasa et al., 1982). Esto se comprueba con lo dicho por Lazarus y Folkman (1986), quienes mencionan que el afrontamiento está formado por ciertos pensamientos y conductas que se ejecutan en respuesta a situaciones estresantes específicas. La relación entre estrés y afrontamiento es recíproca debido a que las acciones que realiza una persona para afrontar un problema afectan la valoración del mismo y la manera subsecuente de hacerle frente.

La resiliencia desde la orientación psicológica propone trabajar ya no sobre los factores de riesgo, sino sobre la capacidad para afrontarlos, poniendo en juego sus capacidades individuales pero sin olvidar que la enfermedad está presente en el paciente. Por lo mismo, se debe tomar en cuenta que éste es un proceso en constante movimiento ya que interactúan la parte física y emocional. La orientación psicológica intenta cambiar la forma en que se percibe al ser humano: de un modelo de riesgo basado en las necesidades y la enfermedad, se plantea ahora un modelo de prevención y promoción basado en las potencialidades y los recursos que el ser humano tiene en sí mismo y a su alrededor (Vanistendael, 1998). 
Como orientadores psicológicos se deben promover las fortalezas y los aspectos positivos del individuo para reducir los factores de riesgo y las fuentes de estrés, sin dejar de lado los altibajos de la enfermedad que están presentes como limitante tanto para el paciente como para el mismo orientador.

Es por esto que se propone dar mayor importancia a la prevención en los diferentes contextos y etapas del desarrollo generando en la población estilos de vida saludables.

Para concluir, es esencial marcar que el cambio de enfoque de la enfermedad crónica que asume el

\section{REFERENCIAS}

Aley, K. (2002). Developmental Approach to Pediatric Transplantation. Progress in Transplantation, 12, 2, 86-91.

Bennet, D. (1994). Depression among Children with Chronic Medical Problems: A Meta-analysis. Journal of Pediatric Psychology, 19, 149-169.

Bradford, R. (1997). Children, Families and Chronic Disease. London: Routledge.

Bradley, G.W. (1993). Disease, diagnosis and decisions. Nueva York: John Wiley \& Sons.

Calhoun, L. \& Tedeschi, R. (2006). The Handbook of Posttraumatic Growth: Research and Practice. Mahwah, NJ: Lawrence Erlbaum Associates.

Cyrulnik, B. (2002). El murmullo de los fantasmas. Madrid: Gedisa.

Eiser, C. (1996). Helping the Child with Chronic Disease: Themes and Directions. Clinical Child Psychology and Psychiatry, 1, 4, 551-561.

Fraser, C. (1997). La Resiliencia. Revista Latinoamericana de Psicología, 23, 2, 14-24.

Garralda, M. (1994). Chronic Physical Illness and Emotional Disorder in Childhood. British Journal of Psychiatry, 164, 8-10.

Grotberg, E. (1997). La Resiliencia en Acción. Trabajo presentado en el Seminario Internacional sobre Aplicación del Concepto de Resiliencia en Proyectos Sociales. Universidad Nacional de Lanas: Fundación Van Leer.

Kern, E. \& Moreno, B. (2007). Resiliencia en Niños Enfermos Crónicos: Aspectos Teóricos. Psicologia em Estudo, 12, 1, 81-86. abordaje de la resiliencia, no significa que se dejen de considerar los problemas que pueden causar tal situación, sin embargo, el planteamiento pretende una visión optimista y esperanzadora para el desarrollo de los enfermos crónicos, quienes pueden desarrollar una visión más alentadora, de aprendizaje y cambio de hábitos para poder convivir con el padecimiento que desde el diagnóstico forma parte de su adaptación social y estilo de vida.

Kobasa, S., Maddi, S. \& Kahn, S. (1982). Hardiness and Health: A Prospective Study. Journal of Personality and Social Psychology, 4, 4, 168-177.

Lazarus, R. y Folkman, S. (1986). El Concepto de Afrontamiento en Estrés y Procesos Cognitivos. Barcelona: Martínez Roca.

Martorelli, A. y Mustaca, A. (2004). Psicología positiva, Salud y Enfermos Renales Crónicos. Revista de Nefrología, Diálisis y Transplantes, 24, 3, 99-104.

Masten, A. \& Powell, J. (2003). A Resilience Framework for Research, Policy, and Practice. En S. S. Luthar, (Ed.), Resilience and vulnerability: Adaptation in the context of childhood adversities. (pp. 1-29). Cambridge: University Press.

Midence, K. (1994). The Effects of Chronic Illness on Children and their Families: An Overview. Genetic, Social \& General Psychology Monographs, 120, 311-316.

Organización Mundial de la Salud (OMS) (2001). Boletín de la Organización Mundial de la Salud. Revista Internacional de Salud Pública.

Perrin, J. \& Shonkoff, J. (2000). Developmental Disabilities and Chronic Illness: An Overview. En R. E. Behrman, R. M. Kliegman y H. B. Jenson, (Eds.), Nelson textbook of Pediatrics. (pp. 452-464). Philadelphia: W. B. Saunders.

Place, M., Reynolds, J., Cousins, A. \& O'Neill, S. (2002). Developing a Resilience Package for Vulnerable Children. Child and Adolescent Mental Health, 7, 4, 162-167.

Rogers, C. (1997). Psicoterapia centrada en el cliente. Barcelona: Paidós. 
Seligman, M. \& Csikszentmihalyi, M. (2000). Positive psychology. An Introduction. American Psychologist, 55, 1, 5-14.

Suárez Ojeda, E. \& Melillo, A. (2001). Resiliencia: Descubriendo las propias fortalezas. Buenos Aires: Paidós.

Szarazgat, D. y Glaz, C. (2006). Resiliencia y Aprendizaje en Sectores Populares. Revista Iberoamericana de Educación, 40, 3, 1-6.

Vanistendael, S. (1998). Cómo crecer superando los percances. Resiliencia: Capitalizar las Fuerzas del Individuo. Colección Cuadernos del BICE. Ginebra: Oficina Internacional Católica de la Infancia.

Vera Poseck, B., Carbelo, B. \& Vecina, M. (2006). La experiencia traumática desde la Psicología Positiva:
Resiliencia y crecimiento postraumático. Papeles del Psicólogo, 27, 1, 40-49.

Yates, T., Egeland, B. y Sroufe, A. (2003). Rethinking resilience: A Developmental Process Perspective. En S. S. Luthar, (Ed.), Resilience and vulnerability: Adaptation in the context of childhood adversities. (pp. 243-266). Cambridge: University Press.

Yates, T. \& Zelazo, L. (2003). Research on Resilience: An Integrative Review. En S. S. Luthar, (Ed.), Resilience and vulnerability: Adaptation in the context of childhood adversities. (pp. 510-549). Cambridge: University Press. 